\title{
Ser y estar en la profesión: la enseñanza en grado de los aspectos deontológicos de la traducción y la interpretación
}

\section{How Professionals Become Professional: Building Ethics into Translation and Interpretation Undergraduate Training}

\author{
Elena PALACIO ALONSO \\ Universidad de Salamanca \\ elenapalacio@usal.es
}

Recibido: julio 2018. Revisado: septiembre 2018. Aceptado: octubre 2018.

Resumen: Nuestro objetivo es reflexionar sobre la interiorización del saber ser profesional y el saber estar en la profesión. Para ello, partimos de la premisa de que las cuestiones deontológicas complementan y refuerzan el aprendizaje de toda profesión. En primer lugar, plantearemos la necesidad de tratar de forma sistemática en la fase formativa los aspectos éticos y deontológicos de traductores e intérpretes en los grados universitarios españoles. A continuación, analizaremos el posible abordaje de estas cuestiones en el aula, en concreto las relativas a la profesión de intérprete de conferencias y partiendo del modelo de Salamanca. En definitiva, consideramos que la responsabilidad de los profesionales lingüísticos va más allá del cliente para alcanzar a la colectividad y a la comunidad en la era de la globalización, donde la traducción (oral o escrita) es, más que nunca, el idioma universal del que toda sociedad se sirve para avanzar y consolidarse. 
Palabras clave: competencia profesional; deontología; ética profesional; formación; interpretación de conferencias.

\begin{abstract}
This paper aims to reflect on how students become professionals and come to embrace their professional community. To this end, we start from the premise of ethics as a way to complement and reinforce learning in any given profession. Firstly, we will elaborate on the need to build professional ethics into translation and interpretation undergraduate training in Spain. We will then examine how ethics can be addressed in the classroom, with a focus on conference interpretation and based on the Salamanca model. In short, we believe that language professionals have a responsibility not only to their client, but also to their colleagues and ultimately to the community, this being the era of globalisation where translation -spoken and written- is, now more than ever, the universal language that all societies rely on to thrive and grow stronger.
\end{abstract}

Key words: professional skills; ethics; professional ethics; training; conference interpretation.

\title{
1. INTRODUCCIÓN
}

Hay una dimensión ética en todo lo que hacemos y una dimensión deontológica cuando, además, vivimos de lo que hacemos y ponemos nuestros conocimientos y habilidades al servicio de la comunidad. Así, las profesiones generan una serie de obligaciones y derechos específicos en quienes las ejercen, lo cual es lógico ya que vivimos constitutivamente en sociedad y nuestra ética singular y nuestra ética profesional no pueden separarse de los otros. Por otro lado, si bien la ética profesional es universal e indisociable de cualquier profesional, la deontología es plural, ya que existe una específica para cada colectivo, de modo que cada profesión ha de cumplir con unas determinadas pautas sociales para ser legítima y gozar de reconocimiento. $Y$ aunque cada profesional le imprima individualidad y particularidad a su labor, la profesión se ejerce de forma colectiva y es el collegium el que determina las normas y obligaciones de sus miembros. Así, su deontología «queda plasmada en un texto escrito aprobado y refrendado por el colectivo profesional del que se trata» (Hortal 2007, 198).

Por consiguiente, defendemos la estrecha relación que existe entre la interiorización de las cuestiones deontológicas y el correcto ejercicio profesional. Creemos que la incorporación generalizada de las cuestiones profesionales a los planes de estudios en Traducción e Interpretación (Tel) en España tendrá una repercusión muy positiva en la profesión -en su correcto ejercicio, en su visibilidad y en su percepción o estatus social-, permitiendo la regeneración del mercado y mejorando la imagen que proyecta en el público general.

Elena PALACIO ALONSO

Ser y estar en la profesión: la enseñanza en grado de los aspectos deontológicos de la traducción y la interpretación
CLINA

vol. 4-2, December 2018, 47-63

elSSN: 2444-1961

Ediciones Universidad de Salamanca - CC BY-NC-ND 


\section{LA FORMACIÓN DE PROFESIONALES COMPETENTES}

A nuestro entender, desde las facultades no podemos obviar las dimensiones éticas presentes en toda ciencia, en toda profesión y, por qué no decirlo, en la propia enseñanza. Sin embargo, tanto en la cultura universitaria como en sus planes de estudios, la enseñanza de las competencias cognitivas y técnicas siempre tuvo un papel preponderante en nuestro país hasta la introducción específica de las competencias éticas y sociales en los programas académicos con el Espacio Europeo de Educación Superior (EEES). Hasta entonces, esta visión más tecnicista de las profesiones llevó a las instituciones de educación superior a atribuir un «menor rango» a las competencias éticas de los profesionales, «incluso presuponiendo que se aprenderían directamente con el propio ejercicio de la profesión» (García López et al. 2009, 202).

Además, tal y como apuntan autores como García Benítez (2006) y Ana Hirsch (2014), los profesionales actuales necesitan una preparación técnica que es condición sine qua non para realizar su trabajo. No obstante, la tecnificación de la profesión puede suponer un obstáculo para el profesional ya que «diluye» su responsabilidad ética. Dicho de otro modo, el trabajador pasa a ser un mero agente técnico instrumentalizado para obtener los resultados que otros desean, pero al mismo tiempo le convierte en una pieza fácilmente sustituible al realizar una función mecánica.

La formación deontológica en el contexto universitario es una demanda inaplazable y absolutamente prioritaria para que el alumno, tras más de quince años de aprendizaje constante en el marco de la educación reglada y, ya en su última fase, preparándose para ingresar en el mercado profesional, pudiera adquirir las herramientas necesarias para realizar esa transición de la manera más provechosa posible tanto para él como para la sociedad destinataria de sus servicios. Pero no pretendemos pecar de ingenuos, pues somos conscientes, al igual que Crisol-Moya y Romero López, de que «[l] a presencia de una materia de ética profesional en los currículos pedagógicos [...] no va a llenar el vacío por sí sola; pero sin ella, [...] [n]i siquiera se podrá encarar debidamente el problema central de toda formación humana en nuestros días, el relativismo moral» $(2014,26)$. Efectivamente, la cuestión de la ética profesional no es opinable y la deontología pone a nuestra disposición las normas para encauzarla.

¿Qué tipo de profesionales queremos que salgan de nuestras aulas? ¿Cómo queremos que sean los futuros traductores e intérpretes? Ambas preguntas, en absoluto retóricas, nos pueden guiar a la hora de calibrar la pertinencia de las cuestiones deontológicas en fase formativa. Consideramos que la universidad dota a la sociedad de capital humano, y este hecho fundamenta una concepción necesariamente copulativa y sinérgica de la relación universidad-sociedad.

Concretamente, la formación universitaria de grado debe ofrecer al alumnado una formación profesionalizante: competencias, usos, habilidades y conocimientos que le habilitan para realizar una actividad especializada a la que no se puede dedicar

Elena PALACIO ALONSO

Ser y estar en la profesión: la enseñanza en grado de los aspectos deontológicos de la traducción y la interpretación
CLINA

vol. 4-2, December 2018, 47-63

elSSN: 2444-1961

Ediciones Universidad de Salamanca - CC BY-NC-ND 
cualquiera. Por lo tanto, entendemos que el título o comprobante de la capacitación profesional, avala a su portador para proporcionar a la sociedad una serie de bienes y servicios a cambio de una retribución digna que constituye su medio de vida. No obstante, creemos igualmente que la universidad no es únicamente un entorno de capacitación profesional, sino que también educa en valores profesionales y, por ende, ciudadanos. Así, consideramos que nuestro objetivo principal como docentes es formar a sujetos competentes, dotados de destrezas cognitivas y reflexivas, por supuesto, pero también éticas y cívicas (Bicocca-Gino 2017) si realmente queremos que nuestros egresados resulten funcionales en la sociedad. Dicho de otro modo,

[...] formar buenos profesionales desde la Universidad significa ayudar al estudiante a que tome conciencia de que quiere ser, por ejemplo, abogado y al mismo tiempo de qué tipo de abogado quiere ser, de forma que se plantee una doble reflexión: profesional y personal de manera conjunta, que encajaría en una nueva didáctica universitaria con el objetivo de formar ciudadanos con una profesión concreta (Esteban, 2004, 56).

Sin ir más lejos, la propia OCDE entiende las competencias como la capacidad del individuo para responder a demandas complejas y ejecutar correctamente tareas diversas, lo que supone a su vez «una combinación de habilidades prácticas, conocimientos, motivación, valores, actitudes, emociones y otros componentes sociales y de comportamiento que se movilizan conjuntamente para lograr una acción eficaz» (García García 2010, 35).

En este sentido, Bolívar (2005) apunta a una sencilla pero eficaz tríada competencial en fase formativa para alcanzar la capacitación integral de los graduados universitarios:

a) conocimientos especializados del campo en cuestión,

b habilidades técnicas de actuación y

c) un marco de conducta en la actuación profesional (Bolívar 2005, 95).

Creemos que a esta tríada debemos atribuirle además un carácter necesariamente combinatorio o contributivo, imperativamente aglutinador. Así, en primer lugar, el alumno debe saber, es decir, debe contar con las competencias cognitivas básicas como dominar su lengua materna y sus lenguas de trabajo, capacidad de redactar, de análisis y de síntesis, etc. En segundo lugar, el saber hacer remite a las competencias técnicas, sean estas traductológicas y/o interpretativas y, por último, el alumno debe entrar en contacto con la ética profesional y con la deontología propia de su gremio para saber ser profesional haciendo gala de unos valores y unas actitudes respetuosas que dignifiquen su profesión y para saber estar en la profesión, es decir, adquirir unas habilidades sociales, unas capacidades de interacción y colaboración

Elena PALACIO ALONSO

Ser y estar en la profesión: la enseñanza en grado de los aspectos deontológicos de la traducción y la interpretación
CLINA

vol. 4-2, December 2018, 47-63

elSSN: 2444-1961

Ediciones Universidad de Salamanca - CC BY-NC-ND 
con personas e instituciones que le permiten entender la comunidad como algo propio y tomar conciencia de su pertenencia a ella. En definitiva, esta «eticidad», como la designan Casares García, Carmona Orantes y Martínez-Rodríguez (2010), hace referencia al conjunto de «principios, rasgos y convicciones que adquieren los estudiantes de manera general para la sociedad y singularmente para cada una de las familias profesionales» $(2010,11)$. Kalina (2015), refrenda esta misma línea de pensamiento al considerar estos principios como la vara de medir el comportamiento profesional que, por un lado, tiene en cuenta los valores que motivan la acción del trabajador y, por otro, el efecto que causa esa acción en los usuarios de su servicio.

Tal y como apuntan García López et al., «no podemos dejar al albur de cada profesional que llegue de manera intuitiva al ejercicio responsable, justo y digno de su trabajo, sin haberle dado previamente nociones, herramientas y estilos que tomar de referencia» $(2009,202)$. La ausencia de esta formación aboca al recién graduado a un learning by doing potencialmente peligroso que permite equivocarse muchas veces antes de dar respuestas deontológicamente defendibles y que, además, estas dependan de la ética individual.

En efecto, el comportamiento profesional -sus actitudes y valores- no es innato al individuo. Por el contrario, creemos que el componente ético indispensable en toda actuación profesional es fruto de un proceso individual que puede y debe enseñarse en fase formativa. Para ello, coincidimos con Drugan y Megone (2011) cuando afirman que el objetivo de enseñar los aspectos éticos y deontológicos en el aula es permitir que los estudiantes desarrollen el buen juicio (good judgement) necesario para identificar los posibles problemas éticos en el momento en el que surgen en una determinada circunstancia, para analizar cómo estos problemas moldearán sus acciones y, por último, para elegir una manera de actuar que constituya una respuesta efectiva en esa circunstancia concreta.

Cuando educamos, claramente estamos, de una forma u otra, imponiendo ideas y prácticas sobre un sujeto al cual inducimos a actuar de una determinada forma que no necesariamente es espontánea o natural para él. La manera de justificar este proceso es la ulterior capacidad de este sujeto de cuestionar o repensar lo que se le ha enseñado. El EEES, y de manera particularmente específica el MEC (Marco Europeo de Competencias) describe la competencia en términos de responsabilidad y autonomía. De este modo, si pensamos en la educación para la autonomía, creemos que esta se fundamenta en establecer unos patrones de trabajo para equipar al individuo con la capacidad de repensar y volver a lo enseñado para cuestionarse su proceso educativo e incluso para revelarse contra lo aprendido en el caso de que no estén de acuerdo. Dicho de otro modo, capacitamos al alumno para que evite el mero adoctrinamiento y automatice un proceso de profesionalización basado en el conocimiento, la acción y la reflexión.

Elena PALACIO ALONSO

Ser y estar en la profesión: la enseñanza en grado de los aspectos deontológicos de la traducción y la interpretación
CLINA

vol. 4-2, December 2018, 47-63

elSSN: 2444-1961

Ediciones Universidad de Salamanca - CC BY-NC-ND 


\section{EL ABORDAJE DE LAS CUESTIONES DEONTOLÓGICAS EN GRADO: EL EJEMPLO DE SALAMANCA}

A continuación, pasamos a exponer de manera sucinta lo que, a nuestro entender, podría constituir la hoja de ruta en el proceso de enseñanza y aprendizaje de las cuestiones éticas y deontológicas en los grados universitarios españoles en Tel. Para ello, hemos tomado como referencia el andamiaje metodológico y estructural de la asignatura Aspectos deontológicos de la traducción y la interpretación, de carácter obligatorio y con una carga de 4,5 ECTS, incluida en el grado en Traducción e Interpretación de la Universidad de Salamanca. Esta asignatura se imparte desde el curso 2010-2011 y, hasta la fecha, no hemos encontrado una materia equivalente en ningún plan de estudios de otro grado español en Tel. Por el momento, los resultados preliminares de las encuestas de satisfacción remitidas a los alumnos desde el curso 2015-2016 avalan la pertinencia de su inclusión en el plan de estudios y un alto grado de utilidad, al tiempo que nos han permitido mejorar su impartición.

El objetivo de esta asignatura es acercar al alumno a la realidad profesional y capacitarle para trabajar con honradez, reflexionar sobre sus funciones y responsabilidades y desarrollar su propia conciencia profesional en beneficio de las dos comunidades que se ponen en contacto a raíz de su mediación lingüística y cultural.

Somos conscientes de que enseñar al futuro profesional a ser congruente y capaz de defender sus posturas, elecciones o decisiones en una situación real sin dañar la imagen del colectivo ni la suya propia no puede hacerse en un único cuatrimestre. No queremos ni podemos arrinconar las cuestiones éticas y deontológicas a una única asignatura y confiar en que sea suficiente, por eso es tan necesario que «todo acto de enseñanza sea intrínsecamente ético» (Crisol-Moya y Romero López 2014, 27). El docente enseña con sus conocimientos, pero también con su ejemplo en todas y cada una de las asignaturas que se imparten en un grado universitario. Así, las acciones del profesor y, en última instancia, su identidad ética, desempeñan un papel importante en el desarrollo de la identidad profesional y ética de sus alumnos y futuros profesionales.

\subsection{El andamiaje teórico: las herramientas conceptuales}

En un primer momento, el docente deberá compendiar una base teórica pertinente, ejemplificada y adaptada para que el alumno se familiarice con la terminología y los conceptos esenciales en las sesiones plenarias. A todo ello, nosotros le añadimos una necesaria aproximación a los códigos deontológicos nacionales que atañen a los intérpretes de conferencia: algunos de ellos genéricos para traductores e intérpretes como el de ASETRAD (Asociación Española de Traductores, Correctores e Intérpretes) y otros específicos como el de AICE (Asociación de Intérpretes de Conferencia de

Elena PALACIO ALONSO

Ser y estar en la profesión: la enseñanza en grado de los aspectos deontológicos de la traducción y la interpretación
CLINA

vol. 4-2, December 2018, 47-63

elSSN: 2444-1961

Ediciones Universidad de Salamanca - CC BY-NC-ND 
España) o el de Espaiic (Asociación de los Miembros de la Asociación Internacional de Intérpretes de Conferencia en España).

En el caso de la traducción y la interpretación, son las asociaciones profesionales quienes elaboran estos códigos que, para autores como Jover Olmeda y Ruiz Corbella (2013), son los encargados de regular conductas, pero también de constituirlas. En otras palabras, un colectivo genera un determinado ethos o identidad profesional aplicando los principios y normas reguladoras que dimanan de los códigos. De este modo, en el aula podemos abordar los códigos como herramientas de socialización, pues redundan en un conjunto de valores compartidos que, en última instancia, dotan de identidad al colectivo.

Llegados a este punto, cobra especial importancia la labor docente, que consistirá en que el alumno llegue a saber interpretar un código y dotarle de flexibilidad y dinamismo, ya que, como explican Drugan y Megone (2011), en el ejercicio profesional siempre pueden darse situaciones en las cuales entren en conflicto varios elementos de dicho código o bien que ni siquiera se reflejen o compendien en el mismo. Dicho de otro modo, los principios deontológicos:

[...] son líneas trazadoras que indican qué es admisible o inadmisible desde un punto de vista moral. Suministran una solidez que la intuición moral, por sí sola, no tiene. Sin embargo, no basta con los principios, con las teorías éticas o con los códigos deontológicos. Hay que someter los principios y las teorías a la prueba de la realidad. Hay que bajar los conflictos desde los valores universales hasta las prácticas concretas social y profesionalmente relevantes (Kwane en Linde Navas 2009, 56).

La deontología es una ética normativa aplicada a una profesión en concreto. De hecho, junto con la legislación, ambos elementos reflejan la heteronomía kantiana en el grado de exigencia profesional (Altisent Trota, Gállego Royo y Delgado Marroquín 2014), ya que constituyen mandatos externos al individuo, normas de obligado cumplimiento impuestas por el colegio profesional y por el Estado respectivamente. Por este motivo, la deontología puede ser percibida por el alumno como rígida, poco flexible y poco adaptada a su manera de concebir el mundo desde el punto de vista moral o de la ética personal (la autonomía en el grado de exigencia profesional; ver Figura 1).

Por lo tanto, es necesario que propiciemos la interiorización de las diferencias entre la ética personal, la ética profesional y su enfoque teleológico y la propia deontología recogida en los códigos para poder aplicar la norma con cierta flexibilidad atendiendo al contexto y a la circunstancia. Con una base teórica y conceptual que contextualice su actuación, el alumno podrá evitar contradicciones flagrantes entre su ética personal y profesional y el conjunto de exigencias externas cuyo cumplimiento está bajo la supervisión del correspondiente gremio profesional.

Elena PALACIO ALONSO

Ser y estar en la profesión: la enseñanza en grado de los aspectos deontológicos de la traducción y la interpretación
CLINA

vol. 4-2, December 2018, 47-63

elSSN: 2444-1961

Ediciones Universidad de Salamanca - CC BY-NC-ND 


\section{Niveles de exigencia profesional}

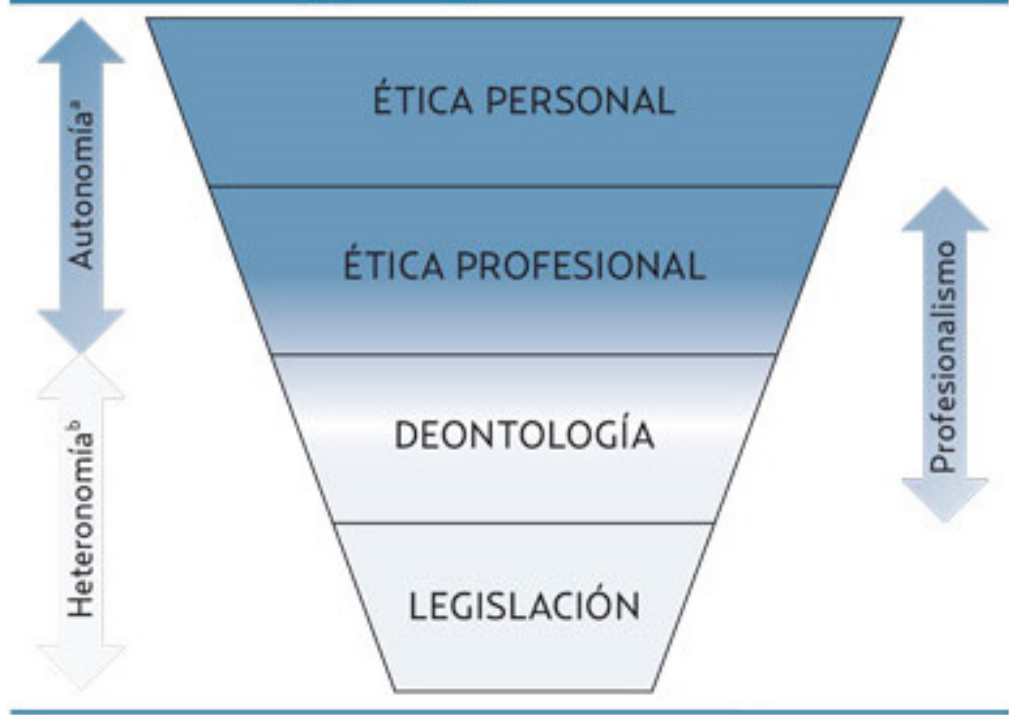

"Autonomía: del griego outo: «por si mismon; y nomo: «regla, norma, orden».

'Heteronomia: del griego hetero: wel otro, distinton; y nomo: uregla, norma, ordens.

Figura 1: Niveles de exigencia profesional (Altisent Trota, Gállego Royo y Delgado Marroquín 2014, 654).

Por último, es necesario abordar el marco legal que regula nuestra actividad profesional para desarrollar otras competencias adyacentes al desempeño profesional y al comportamiento profesional, como pueden ser las obligaciones fiscales y con los organismos de previsión social, relación con las empresas e instituciones que pueden ser potenciales clientes, información sobre contratos y tarifas en el libre mercado, estrategias de negociación, etc.

Es importante destacar que el conocimiento de la norma no garantiza su aplicación o su cumplimiento y, por lo tanto, no podemos concebir el proceso de enseñanzaaprendizaje de las cuestiones éticas y deontológicas como una mera sustitución del hábito moral por el seguimiento ciego de unas normas establecidas por las asociaciones profesionales en los códigos deontológicos. Sin embargo, no desdeñamos la utilidad de estos últimos ni nos decantamos tan categóricamente por los propios valores y la integridad del individuo como Baker y Maier cuando afirman que «[r]eflecting critically on ethical behaviour means examining one's own values, becoming more aware of them, and assessing them critically. It is ultimately a question of personal integrity, not skill in following a prescribed set of rules» $(2011,4)$, entre otros motivos, porque es muy posible -como hemos señalado más arriba- que dicha integridad moral puede contravenir la propia ética profesional, o incluso la deontología concreta de su profesión. 
Para ello, creemos necesaria la casuística real y/o simulada que fomente el debate y la aplicación práctica de estos principios que, como bien explica Kalina (2015), se reflejan en nuestro comportamiento profesional antes, durante y después del encargo. Para Baker y Maier, «[b]uilding ethics into the curriculum means opening up a space for critical reflection, training students to think through the consequences of their behaviour, rather than telling them what is right or wrong per se» $(2011$, 4). Por un lado, compartimos la opinión de estas autoras cuando abogan por la reflexión crítica del alumno. Sin embargo, por otro lado, también consideramos que el conocimiento «es un requisito fundamental de toda elección y acción racionales» (Linde Navas 2009, 38), y por eso es tan importante una fundamentación teórica que permita una toma de decisiones informada. A nuestro entender, ambos elementos tienen peso en la formación y han de ser necesariamente complementarios para conseguir la interiorización de actitudes y hábitos virtuosos en la dirección deseada.

Para acercar al alumno a todos estos elementos, defendemos la necesidad de la clase magistral "como un elemento más de la experiencia del aprendizaje» (Linde Navas 2009, 38), puesto que la información y los procedimientos que se derivan de la teoría son igualmente importantes para la interiorización de la ética profesional y del debate posterior.

\subsection{La práctica profesional del docente}

En segundo lugar, creemos que es fundamental sacarle el mayor partido posible a la experiencia de vida de nuestros docentes, ya que constituye el modelo profesional y de socialización del alumno. En este apartado en concreto, nos centraremos en la interpretación por ser nuestro ámbito de especialidad.

Con profesionales en activo implicados en la transmisión de las actitudes y los valores inherentes a toda buena praxis, podemos guiar mejor a las nuevas generaciones en su paso al mundo laboral, supervisando con ellos todo el proceso de actuación profesional, desde el primer contacto con un cliente hasta la emisión y cobro de la factura correspondiente al encargo que hemos realizado para él. Tal y como explica Camilo, «[a] practicing professional is someone who not only has trained to be an interpreter, but who works as one» (2004), hecho que, de por sí, nos ofrece una doble garantía.

A continuación, expondremos brevemente las principales ventajas de contar con profesionales que, a su vez, son intérpretes en activo.

En primer lugar, los intérpretes formadores o practisearchers, como los denomina Boéri (2015), lidian constantemente con una amplia variedad de temas en sus encargos profesionales, lo que les permite emplear material real en el aula al tiempo que comparten con los alumnos sus técnicas de preparación y documentación.

Elena PALACIO ALONSO

Ser y estar en la profesión: la enseñanza en grado de los aspectos deontológicos de la traducción y la interpretación vol. 4-2, December 2018, 47-63

elSSN: 2444-1961

Ediciones Universidad de Salamanca - CC BY-NC-ND 
Asimismo, el formador intérprete conoce de primera mano la evolución de los distintos ámbitos de especialidad en los que trabaja y, por este motivo, experimenta la propia evolución de sus lenguas de trabajo, activas y pasivas, y de sus técnicas.

En tercer lugar, la presencia de profesores-intérpretes en las aulas permite ajustar el contenido de la asignatura a las exigencias y necesidades del mercado real privado, institucional, o incluso ambos si el docente está familiarizado con los dos. De este modo, puede diseñar tanto el temario como la evaluación, de manera que la adquisición de competencias se ajuste a las necesidades de sus posibles contratadores.

Además, Camilo (2004) afirma con rotundidad que los "[s]tudents should have the chance to see and hear professional interpreters" porque la experiencia y el saber hacer se transmiten con más que palabras. Así, evocar y compartir ejemplos, situaciones o incluso errores propios de los que el intérprete-docente ha aprendido facilita la interiorización de una profesión. Y mucho más si permitimos que los alumnos acompañen a los profesionales en encargos reales, por ejemplo, en el marco de prácticas de acompañamiento en cabina. En estos contextos, los alumnos pueden observar no solo el trabajo de sus profesores en cabina, por ejemplo, sino también la relación con todos los actores que intervienen en el proceso, como los propios ponentes, los organizadores, los técnicos o el público. Nuestra experiencia docente a este respecto refuerza la idea de que esta suerte de «apadrinamientos» facilita la interiorización de una profesión que depende en gran medida del propio bagaje vivencial y de la exposición a situaciones y contextos muy variados. En estas experiencias compartidas, se potencia la empatía profesor-alumno y, además, el docente puede adecuar mejor el ritmo formativo en el aula, sabiendo cómo y cuándo introducir un sustento teórico o un determinado ejercicio práctico para desarrollar una destreza concreta.

En quinto lugar, el intérprete-formador trabaja con otros intérpretes, algo que, sin duda, enriquece su sustrato docente pues multiplica exponencialmente el factor vivencial y experimental al compartir las vivencias laborales. En esta línea, las relaciones profesionales pueden conducir a sinergias docentes en forma de charlas, talleres o prácticas con intérpretes invitados que pueden ofrecer puntos de vista sobre la profesión y su ejercicio que enriquezcan la labor del profesor y la socialización gremial del alumno.

Tampoco podemos olvidar que estos intérpretes formadores suponen una plusvalía docente cuando son capaces de acercar al alumno a cuestiones como la gestión del estrés, la resistencia mental a la fatiga y a la rutina en cabina, las dudas sobre la aceptación de un encargo, la disciplina y la necesidad de crearse una buena reputación que fideliza clientes o las ventajas que ofrece el respeto de los códigos deontológicos que, como destaca Camilo (2004), no son ideales abstractos sino una ayuda real para la práctica profesional, ya que constituyen un instrumento para la promoción de la excelencia y, por tanto, de la calidad del servicio.

Por ultimo, consideramos imprescindible «to alert trainees to the ethical implications of behaviour that they might regard as routine, unproblematic, and hence not

Elena PALACIO ALONSO

Ser y estar en la profesión: la enseñanza en grado de los aspectos deontológicos de la traducción y la interpretación
CLINA

vol. 4-2, December 2018, 47-63

elSSN: 2444-1961

Ediciones Universidad de Salamanca - CC BY-NC-ND 
experience as challenging from a moral point of view» (Baker y Maier 2011, 3). Efectivamente, es harto improbable que el joven profesional que entra en el mercado de la interpretación, por ejemplo, perciba como problemática la elección de su domicilio profesional si no sabe que puede incurrir en competencia desleal con intérpretes locales, o que puede enemistarse con agencias con las que colabora habitualmente si entrega a un cliente su tarjeta de visita. Por otra parte, es lógico que el alumno no perciba como un problema ético o una disyuntiva laboral una situación a la cual no se ha expuesto nunca, por eso resulta tan importante que el docente reconozca y conozca esas situaciones y advierta de su existencia en la fase formativa del futuro profesional.

En este sentido, Donovan (2011) añade que los formadores que son al mismo tiempo intérpretes profesionales perciben su trabajo como un «commitment to the upholding of quality, prefessional standards and conditions. It explicitly aims to transmit a certain vision of the profession and is thus closely bound up with socialization» (2011, 121). Además, para esta autora, la percepción de las cuestiones éticas vendría determinada no solo por el nivel de profesionalización, sino también por los procesos de socialización de sus miembros. Esto se debe a que los intérpretes noveles suelen acceder a la profesión a través de la formación en las instituciones de educación superior. De este modo, la formación por parte de intérpretes profesionales asentados y experimentados, dotaría a los alumnos de una identidad profesional bastante sólida y refrendada por las asociaciones profesionales.

Como conclusión, el docente que está en contacto con el mundo profesional porque ejerce -al menos ocasionalmente- la interpretación, se sitúa en una posición privilegiada, con conocimientos constantemente actualizados sobre la profesión que enseña, lo que le habilita para desempeñar un papel bisagra absolutamente esencial para el éxito del proceso de enseñanza-aprendizaje.

\subsection{El aprendizaje basado en la experiencia del alumno}

Confiamos en el aprendizaje basado en la experiencia directa, es decir, en la resolución de problemas reales o simulados que nos ofrecen tanto el aula de interpretación como las prácticas curriculares. Ambos entornos nos permiten tener en cuenta la casuística y la aplicación práctica del conocimiento teórico y constituyen un complemento ideal para contextualizar la asignatura referente a las cuestiones éticas y deontológicas. Como hemos avanzado, este abordaje contribuye a la formación integral del alumno, ya que acerca la teoría a la práctica y conjuga la reflexión con la acción.

La metodología particularmente activa y participativa de este tipo de asignaturas prácticas (tanto las clases de interpretación como las prácticas reales y simuladas que en Salamanca, se enmarcan en la asignatura Modalidades de interpretación) propicia el debate de las cuestiones éticas y deontológicas inherentes a la profesión, pero al

Elena PALACIO ALONSO

Ser y estar en la profesión: la enseñanza en grado de los aspectos deontológicos de la traducción y la interpretación
CLINA

vol. 4-2, December 2018, 47-63

elSSN: 2444-1961

Ediciones Universidad de Salamanca - CC BY-NC-ND 
mismo tiempo también posibilita que el propio alumno se acostumbre a cuestionarse constantemente el ejercicio profesional que realizará en el futuro, ya que ha de encontrar respuestas de manera autónoma -o parcialmente guiada- en un contexto controlado. Entendemos que «[e]s más fácil que los profesionales se rebelen contra los atropellos más graves cuando han adquirido una formación sólida y realista en hábitos morales que potencien los fines internos de la profesión» (Linde Navas 2009, 43), pero para ello abogamos por un abordaje holístico que aúne el conocimiento teórico con la reflexión práctica y, todo ello, tutelado por un principio rector fundado en sensibilizar al alumno sobre las cuestiones deontológicas y de la ética profesional.

En el caso de la asignatura Aspectos deontológicos de la traducción y la interpretación, los alumnos en gran grupo han de identificar el problema (cuándo se incurre en competencia desleal, las implicaciones del domicilio profesional para un intérprete, publicitarse cuando ha sido contractado por una agencia u otro compañero, etc.) y, utilizando los recursos adecuados que les proporcionamos en las sesiones magistrales, aportar posibles soluciones argumentadas y apoyadas tanto en la ética profesional como en los códigos deontológicos que manejamos. Asimismo, el docente desempeña un papel de mediador muy activo, moderando los debates e intentando que el peso de la argumentación recaiga sobre el alumno, limitándose puntualmente a avivar el diálogo con la introducción de puntos de vista novedosos o incluso polémicos.

Como es lógico, este aprendizaje basado en problemas (ABP) únicamente resulta de utilidad si los problemas planteados a los alumnos en el aula describen situaciones reales de la práctica profesional. Tal y como explican Ansoleaga San Antonio y Gómez Gutiérrez, el trabajo del alumno «no se reduce sólo a la búsqueda de la solución, sino también al análisis de su fundamentación, obligando a manejar argumentos de racionalidad respecto al proceder [...]» $(2004,8)$. Dicho de otro modo, «a translator or interpreter must be able to justify a decision (morally) to him or herself as well as those who might question it» (Baker y Maier 2011, 3). Por este motivo, muchos autores, como Linde Navas, también defienden el trabajo en el aula con dilemas reales, ya que estimula entre los alumnos el hábito de deliberar y reflexionar para tomar las decisiones correctas, "considerando siempre los principios y criterios éticos, los valores más universalizables de nuestra cultura, las normas deontológicas que la propia profesión ha generado y las circunstancias contextuales» (2009, 42).

Esta sensibilidad y esta reflexión ética pueden cultivarse en la fase formativa y, desde nuestra experiencia en el aula, creemos que el alumno las interioriza mejor cuando la teoría normativa se aplica a problemas importantes para ellos y se ejemplifica con casuística real extraída tanto de la experiencia del propio profesorado como de la experiencia del propio alumno en prácticas. En definitiva, apostamos por el «aprendizaje profundo» (Linde Navas 2009) frente al superficial que se limita a la memorización puntual de contenidos para aprobar un examen conducente a un título, por ejemplo.

Elena PALACIO ALONSO

Ser y estar en la profesión: la enseñanza en grado de los aspectos deontológicos de la traducción y la interpretación
CLINA

vol. 4-2, December 2018, 47-63

eISSN: 2444-1961

Ediciones Universidad de Salamanca - CC BY-NC-ND 
Podemos pensar que el alumno cuenta ya con el conocimiento teórico de las normas a partir del momento en el que lee un código de conducta profesional, pero las normas son fijas y el contexto que rodea la actuación del futuro profesional no lo es. Por este motivo son tan importantes las prácticas, que aportan al alumno un compendio de experiencias y conocimientos directamente derivados del contacto con otros profesionales y con los clientes de sus servicios que le permiten la adquisición de hábitos virtuosos que garantizan que la actuación es coherente con la reflexión.

Llegados a este punto, creemos necesario reforzar la colaboración y el intercambio de experiencias con los socios de prácticas, lo que permite, por un lado, la revisión constante de los contenidos curriculares y su pertinencia para adecuarlos a las necesidades del mercado y, por otro lado, la «reeducación» del mercado en base a comportamientos más éticos y virtuosos. Por ejemplo, permite hacer ver de manera paulatina a clientes y contratadores que una tarea tan importante como la interpretación no puede realizarse sistemáticamente bajo presión, sin acceso previo a la documentación que manejan los expertos y una remuneración muy cuestionable, pues estos factores propician que dichas interpretaciones, a pesar del valor para la comunidad que las consume, contengan errores e inexactitudes. Estas sinergias, que parten de los convenios de prácticas y que resultan beneficiosas para ambas partes, redundan en la sustitución de ciclos viciosos por virtuosos y nos ayudan a profesionalizar el mercado.

Por último, consideramos que las normas y deberes morales (en este caso, profesionales) no pueden ser meras imposiciones dictadas desde fuera por los códigos de conducta, sino que conviene que sean dictadas y asumidas por el propio individuo. Jones (2016) alerta de la dificultad de inculcar a los alumnos la importancia de aceptar la deontología profesional, por eso creemos que la combinación de elementos racionales y emocionales motiva les puede motivar a actuar correctamente. Las prácticas reales ayudan al traductor o intérprete novel a constatar que ahí fuera hay muchos otros profesionales y usuarios de sus servicios que dependen y confían en su trabajo para, a su vez, desempeñar su propia labor. Sentirse útiles y recibir un feedback de su cliente les aporta a los traductores e intérpretes en formación la motivación extra que, sin duda, marca una diferencia cualitativa en la prestación de cualquier servicio. Lo que queremos decir con esto es que las normas solo pueden ser plenamente éticas para el profesional cuando «él mismo las concibe y asume como propias y buenas, y por ello las obedece» (Bonete en Linde Navas 2009, 43).

\section{CONCLUSIONES}

En primer lugar, quienes tenemos unas responsabilidades educativas y formativas debemos reflexionar sobre el tipo de profesional que sale de nuestras aulas para, en última instancia, posibilitar la reflexión del alumno sobre el tipo de profesional que quiere

Elena PALACIO ALONSO

Ser y estar en la profesión: la enseñanza en grado de los aspectos deontológicos de la traducción y la interpretación
CLINA

vol. 4-2, December 2018, 47-63

elSSN: 2444-1961

Ediciones Universidad de Salamanca - CC BY-NC-ND 
ser. Esta convicción se fundamenta en una concepción necesariamente copulativa y sinérgica de la relación universidad-sociedad. Si la educación superior descuida la formación ética como parte integrante del conocimiento profesional y de su práctica, podemos llegar a cuestionar el papel de la universidad y la calidad de su formación profesionalizante.

Dicho de otro modo, si nos ceñimos a una lógica instrumentalista, por la cual las competencias son el medio para alcanzar el éxito profesional, corremos el riesgo de impedir el desarrollo integral del alumno como profesional y como persona. El EEES hace hincapié en la empleabilidad de los egresados y en la necesidad de un aprendizaje permanente que impida su obsolescencia, -es decir, que el individuo siga resultando atractivo en el mercado laboral. Sin embargo, la universidad ofrece planes de estudio llenos de «saberes expertos de alta utilidad técnica, pero también de alta caducidad temporal» (Bicocca-Gino 2017, 275). En otras palabras, estamos ofreciendo a nuestros alumnos unos contenidos formativos que les proporcionan una serie de destrezas y habilidades que pierden progresivamente su valor. Así, «las competencias que son necesarias hoy ya no lo serán en unos años» $(2017,275)$, a no ser que estas se acompañen de unas competencias éticas y deontológicas que les permitan y conminen a la constante actualización de sus herramientas y conocimientos a lo largo de toda su trayectoria profesional, el aprendizaje permanente.

«[E]l egresado universitario, para ser atractivo a una organización, debe mostrar iniciativa, creatividad y capacidad para establecer relaciones entre el mundo de la comprensión y el de la acción» (Bicocca-Gino 2017, 271). Por eso creemos que el aprendizaje permanente debe partir de un aprendizaje para la vida, donde confluyan la razón teórica (el conocimiento) y la razón práctica (la acción) en el proceso formativo, pero sin descuidar en ningún momento el desarrollo de la razón instrumental (la reflexión) si queremos que el alumnado sea capaz de gestionar la repercusión social de su futura labor profesional.

Por eso creemos que es tan necesario «revitalizar el histórico y necesario papel de la universidad en la formación de una ciudadanía crítica» (Bolívar 2005, 100) si queremos conseguir profesionales igualmente críticos y reivindicativos de unas condiciones laborales dignas; defensores de su actividad profesional al tiempo que se responsabilizan de sus actuaciones en un equilibrio, si no perfecto, sí más armónico, entre los deberes y los derechos de todo profesional.

Así, entendemos que la deontología es pura praxis. Por muy clara que nos parezca sobre el papel, tal y como queda redactada en un código, el dilema, la pregunta o la situación comprometida solo aparecerán realmente durante el propio ejercicio de la profesión. Este es el motivo por el cual necesitamos decidir rápido y con seguridad, habiendo interiorizado ciertos mecanismos de respuesta rápida en fase formativa que impidan la parálisis o la reacción precipitada una vez que nos encontremos confrontados a un problema real y apremiante. A fin de cuentas, la idea es aportar al alumno la

Elena PALACIO ALONSO

Ser y estar en la profesión: la enseñanza en grado de los aspectos deontológicos de la traducción y la interpretación
CLINA

vol. 4-2, December 2018, 47-63

eISSN: 2444-1961

Ediciones Universidad de Salamanca - CC BY-NC-ND 
seguridad de que está capacitado para tomar decisiones y que dichas decisiones no son reprochables porque cuenta con los argumentos éticos y/o deontológicos necesarios para defenderla.

En segundo lugar, el acercamiento del alumno a la conducta profesional puede redundar en el establecimiento de un círculo virtuoso (formación> profesión> mercado> sociedad) que confiera visibilidad al profesional y dote de mayor prestigio social a la profesión, algo que -sin duda- le permitirá reivindicar y mejorar sus condiciones laborales (ver Figura 2). Mediante la formación, el alumno podrá conocer, interiorizar y cumplir sus deberes y, por otro lado, estará en disposición de constituir, expresar y reivindicar unas condiciones laborales y retributivas dignas que dignifiquen la profesión y la doten de un mayor reconocimiento social.

Por otro lado, a falta de un colegio profesional y de un código de conducta unificado para toda la profesión, en España tenemos numerosas asociaciones que actúan como colegios y que funcionan como entidades normativas estableciendo por escrito en sus códigos los deberes y los derechos de los profesionales que los suscriben. En el momento en el que nuestros alumnos, sean socios o no, se avienen motu proprio a unos estándares profesionales compartidos por el colectivo, conseguimos que la profesión sea más fuerte. A fin de cuentas, la armonía profesional es una acción de conjunto y se plasma en la imagen que se crea cada sociedad de un determinado colectivo.

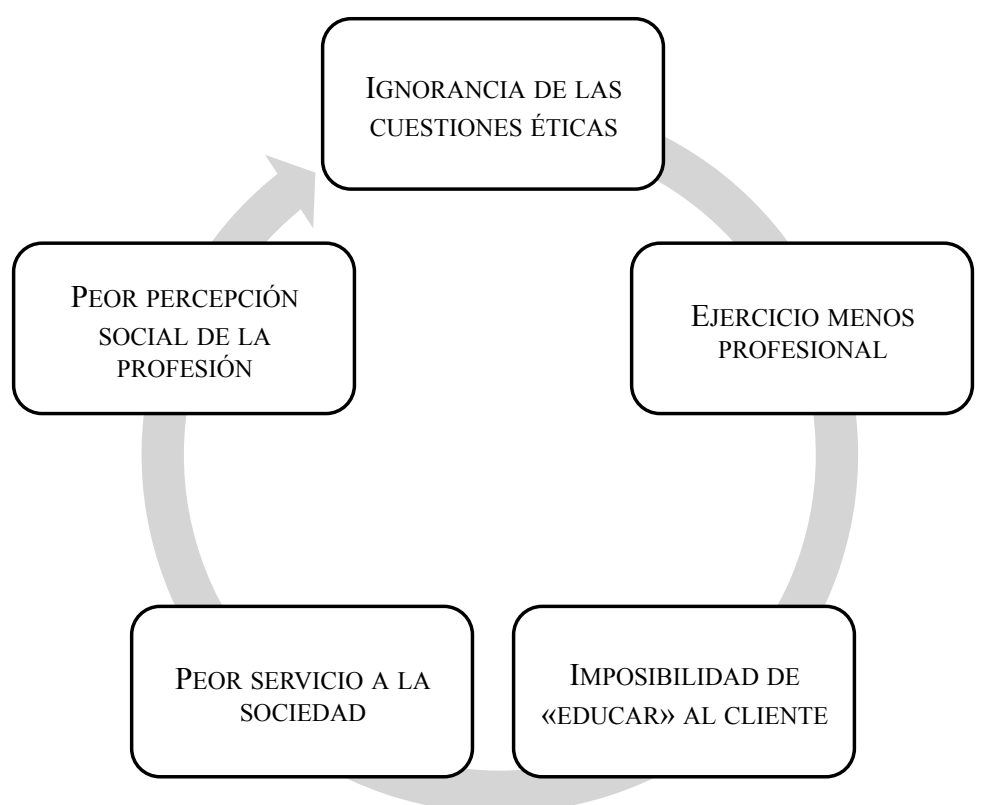

Figura 2: La importancia de las competencias ético-sociales para la formación de profesionales competentes.

Elena PALACIO ALONSO

Ser y estar en la profesión: la enseñanza en grado de los aspectos deontológicos de la traducción y la interpretación vol. 4-2, December 2018, 47-63

elSSN: 2444-1961

Ediciones Universidad de Salamanca - CC BY-NC-ND 
Del mismo modo, un profesional lingüístico competente deja su impronta en el propio mercado, que puede repensarse y mejorar en el momento en el que exige unas condiciones laborales adecuadas que le permiten optimizar el resultado de su trabajo de mediación, es decir, su servicio a la sociedad. En definitiva, la consolidación de una profesión deriva a su vez, y entre otras cosas, de la percepción que la sociedad tiene de quienes la practican.

Por todos estos motivos, creemos que el ser y el estar integrados en el proceso formativo complementan y refuerzan el aprendizaje de la profesión y, también por todo lo expuesto, justificamos su pertinencia y su inclusión en los planes de estudios de los grados españoles en Tel.

\section{BIBLIOGRAFÍA}

Altisent Trota, Rogelio, Alba Gallego Royo y M. ${ }^{a}$ Teresa Delgado Marroquín. 2014. "Los códigos de deontología profesional». AMF 10 (11): 651-658. Fecha de acceso 26 de noviembre de 2018. http://amf-semfyc.com/web/downloader_articuloPDF. php?idart=1376\&id=03NTC-12-14.pdf

ANSOLEAGA SAN ANTONIO, David y Juan Luis GÓMEZ GUTIÉRREZ. 2004. «La reflexión ética en la práctica de la educación social». Comunicado leído en la Universidad de Santiago de Compostela, 2 de octubre de 2004. Fecha de acceso 23 de agosto de 2018. http://www. eduso.net/archivos/Vcongreso/comunicaciones/c44.pdf

BAKER, Mona y Carol MAIER. 2011. «Ethics in Interpreter \& Translator Training: Critical Perspectives». The Interpreter and Translator Trainer 5 (1): 1-14.

BICOCCA-GINO, Rodolfo Mauricio. 2017. «Análisis crítico-filosófico de las potencialidades educativas de la enseñanza basada en competencias». Educación y Educadores 20 (2): 267-281.

BoÉRl, Julie. 2015. «Key Internal Players in the Development of the Interpreting Profession». En The Routledge Handbook of Interpreting, ed. por Holly Mikkelson y Renée Jourdenais. Abingdon: Routledge Handbooks Online, 29-44. Fecha de acceso 21 de octubre de 2018. https://www.routledgehandbooks.com/doi/10.4324/9781315745381.ch2

BOLívAR, Antonio. 2005. «El lugar de la ética profesional en la formación universitaria». Revista Mexicana de Investigación Educativa 10 (24): 93-123.

CAMILO, Silvia. 2004. «Why Trainers Should Be Practicing Conference Interpreters». Ailic.net (sitio web). Fecha de acceso 12 de septiembre de 2018. http://aiic.net/p/1394

CASARES García, Pilar M., Gabriel CaRmona ORANTES y Francisco Miguel MartínezRODRIGUUEZ. 2010. «Valores profesionales en la formación universitaria». Revista electrónica de investigación educativa 12 (SPE): 1-15.

CRISOL-MOYA, Emilio y M. ${ }^{a}$ ROMERO LÓPEZ. 2014. «Práctica docente versus ética docente. Hacia la mejora de la práctica docente a partir de la ética profesional». Journal for Educators, Teachers and Trainers 5 (2): 23-35. Fecha de acceso el 29 de junio de 2017. http://hdl. handle.net/10481/39345

Elena PALACIO ALONSO

Ser y estar en la profesión: la enseñanza en grado de los aspectos deontológicos de la traducción y la interpretación
CLINA

vol. 4-2, December 2018, 47-63

elSSN: 2444-1961

Ediciones Universidad de Salamanca - CC BY-NC-ND 
DONOVAN, Clare. 2011. «Ethics in the Teaching of Conference Interpreting». The Interpreter and Translator TRAINER 5 (1): 109-128. DOI: 10.1080/13556509.2011.10798814

DRUGAN, Joanna y Chris MEGONE. 2011. «Bringing Ethics into Translator Training». The Interpreter and Translator Trainer 5 (1): 183-211. DOI: 10.1080/13556509.2011.10798817

ESTEBAN, Francisco. 2004. Excelentes profesionales y comprometidos ciudadanos. Bilbao: Desclée De Brouwer.

GARCíA BENÍTEZ, Claudia. 2006. «Ética de las profesiones», reseña de Ética general de las profesiones, de Augusto Hortal, Revista de la Educación Superior 35 (137): 127-132.

GARCÍA GARCÍA, Emilio. 2010. «Competencias éticas del profesor y calidad de la educación». Revista Electrónica Interuniversitaria de Formación del Profesorado 13: 29-41. Fecha de acceso 19 de octubre de 2018. http://www.redalyc.org/html/2170/217015570003/

GARCía LÓPEZ, Rafaela, Auxiliadora SALES CIGES, María Odet MOLINER GaRCía y Reina FERRÁNDEZ BARRUECO. 2009. «La formación ética profesional desde la perspectiva del profesorado universitario». Teoría de la Educación. Revista Interuniversitaria 21: 199-221.

HIRSCH ADLER, Ana. 2014. "Ética profesional y empleabilidad: problemas expresados por profesores de universidades en México y en España». Social and Behavioral Sciences 139: 175-181.

HORTAL, Augusto. 2007. Ética profesional de traductores e intérpretes. Bilbao: Desclée De Brouwer.

JONES, Roderick. 2016. «Professional Ethics in Interpreter Training». Fecha de acceso el 15 de junio de 2018. http://www.emcinterpreting.org/node/274?q=system/files/RoderickJonesProfessionalEthics.pdf

JOVER OLMEDA, Gonzalo y Marta RUIz CORBELLA. 2013. «El código deontológico de la profesión docente: evolución y posibilidades». Edetania 43: 113-131.

KALINA, Sylvia. 2015. «Ethical Challenges in Different Interpreting Settings». MonTI 2 (núm. esp.): 63-83. DOI: 10.6035/MonTI.2015.ne2.2.

LINDE NAVAS, Antonio. 2009. "Teorías y procedimientos de educación moral en ética y deontología de la comunicación». Comunicación y sociedad 22 (2): 35-58.

Elena PALACIO ALONSO

Ser y estar en la profesión: la enseñanza en grado de los aspectos deontológicos de la traducción y la interpretación vol. 4-2, December 2018, 47-63

elSSN: 2444-1961

Ediciones Universidad de Salamanca - CC BY-NC-ND 
